\title{
Literature for Amateur Astronomers
}

\author{
By L.S. Kudashkina \& I.L.Andronov \\ Department of Astronomy, Odessa State University \\ T.G.Shevchenko Park, Odessa 270014 Ukraine
}

The experience of working with amateur astronomers in the countries of the Commonwealth of Independent States and in Ukraine shows a noticeable lack of literature, especially educational and methodological. The amateurs, possessing an observational base, do not know what best to observe at a given moment, and those, who are not yet ready for practical work in astronomy, do not know how to be prepared.

A series of brochures under the title "The Atlas of Amateur Astronomy"has been prepared, which pursues the purpose of delivering to amateurs a minimum of the necessary information on the following items:

1. Popular scientific reviews (lectures) on various directions in astronomy and astrophysics.

2. Methodological articles on the bases of observations and their processing.

3. Programs of observations, finding charts of variable stars, short information on comets, meteor showers etc.

4. Help material (tables, ephemerides, items of information from the General Catalogue of Variable Stars etc.).

5. Observations made by the amateurs themselves.

Five issues of "The Atlas of Amateur Astronomy" have been published. Together they contain information on about 60 objects, for which finding charts and comparison stars are given.

Part I contains the introductory articles, description of a structure of the atlas, which is repeated in the other issues, finding charts for 20 variable stars, recommendations for observations and the table of Julian dates from 1980 till 1995 (the atlas was issued in 1990).

Part II contains the first lecture from a cycle "Variety in the world of variable stars" on a theme "Long-period variables". In this part the finding charts with comparison stars for 30 variables are given. Instruction is also given here on how to make observations and how to reduce moments of time to the center of the Sun for short-period stars.

Part III contains a lecture on a theme "Binary stars" (general and theoretical aspects). In this part only two finding charts (for the stars $\mathrm{R}$ Tri and RU Cam) are shown, but the standard NPS (North Polar Sequence) is given and a lot of help material, requested by amateur astronomers, e.g. the names of variable stars, Greek and Latin alphabets, list of constellations. Also, in the "short communications" there are placed observations of the Mira-type star R Aql and a drawing of sunspots.

Part IV contains a continuation of the lecture about binary stars, namely "Determination of the physical characteristics of eclipsing binary stars". This issue also contains information about the 2nd Congress of the Astronomical Society of the Former Soviet Union in Moscow. The most interesting reports are briefly described. Also in part IV are placed the electrophotometric standards IC 4665 and Coma Berenices. A finding chart of the Mira-type star R Cas is given, and in the short communications - observations of the bright bolide above Saratov city.

Part V was issued in 1996 after a long gap (parts II-IV are dated 1991). It contains a lecture from the same cycle on a theme "Supernova stars". It is opened by an article about the Crab Nebula. The detailed information about the bright supernovae 1987 
and 1993 is also included. Finding charts for 4 variable stars are placed. In the short communications, observations of the supernova 1993 in M81 are indicated.

Another series of publications has been prepared by I.L.Andronov and L.L.Chinarova for the amateurs who are members of the section of Astronomy of the Small Academy of Sciences "Prometheus" working at the Odessa Regional Station for Young technicians. This "Academy" gathers scholars interested in different branches of science - astronomy, mathematics, physics, chemistry, biology, ecology, PC programming, air plane- and automodeling, humanitarian sciences et al. It works under the supervision of the associate and full professors from the Odessa State University and sometimes other institutes. Every year competition conferences are organized starting in individual schools, progressing through regional heats with the final covering the whole of Odessa State. The winners are recommended to all-Ukrainian and international conferences. The best scientific works made by scholars are being published in the annual volume of the proceedings booklet "Pervye shagi"("First steps"). These publications are of course not so important for science as the professional journals, but they are really first steps and some of the authors improve these contributions and resubmit articles to more "serious" journals. In the section of astronomy this takes place usually if a variable star is observed visually or photographically. One may note that the Sky Patrol plate collection of the Astronomical Observatory of the Odessa State University contains about 100,000 negatives and thus is the third in the world after Harvard (USA) and Sonneberg (Germany) and attracts attention of professionals and amateurs from many countries. Such articles were published in "Variable Stars", "Astronomical Circular"(Russia), Information Bulletin on Variable Stars (Hungary), Bulletin de l'AFOEV (France), "The Astronomer"(United Kingdom), "Variable Star Net (VSNET)" (Japan), “Odessa Astronomical Publications"(Ukraine). Among the most active observers making not only first but many other subsequent steps are A.V.Halevin, V.I.Marsakova, Yu.V.Beletskij, K.A.Antoniuk.

For amateurs, summer schools are organized, and also a "correspondence course" which was the only one in the former Soviet Union and was initiated by Professor V. P. Tsessevich (1907-1983) in 1977. Now it is the only one in the Ukraine. In Russia a similar course started in Ekaterinburg. For the amateurs and students several years ago a series of booklets was published in Odessa by I.L.Andronov. Among them: "Visual and photographic observations of variable stars" $(1991,84 \mathrm{pp}$.), "Structure and evolution of variable stars" (part 1, 1991, 86pp, Part 2, 1992, co-author L.L.Chinarova) and 16 smaller ones.

Some popular articles by I.L.Andronov were published in the journals "Priroda" (Moscow, 1987), "Die Sterne" (Leipzig, 1988, 1989), "L’Astronomia" (Milan, 1995), "Koszmos" (Prague, 1996).

Popular articles on various fields of Astronomy in the Ukrainian language are published in "Short astronomical calendar" issued in Kiev.

We hope that our attempts contribute a little to the popularization of astronomy in our country and will be happy if this could help somebody "to find his own star". 